\title{
Exact Multisensor Dynamic Bias Estimation with Local Tracks
}

\author{
X. Lin and Y. Bar-Shalom \\ Dept. of ECE \\ University of Connecticut \\ Storrs, CT 06269-2157, U.S.A. \\ \{xdlin,ybs\}@ee.uconn.edu
}

\author{
T. Kirubarajan \\ ECE Department \\ McMaster University \\ Hamilton, Ontario, Canada L8S 4K1 \\ kiruba@mcmaster.ca
}

\begin{abstract}
This paper provides the exact solution of multiple sensor bias estimation problem based on local tracks. It is shown that the bias estimate can be obtained dynamically using the outputs of the local (biased) state estimators. This is accomplished by manipulating the local state estimates such that they yield pseudomeasurements of the sensor biases with additive noises that are zero-mean, white and with easily calculated covariances. These results allow evaluation of the Cramer-Rao Lower Bound (CRLB) on the covariance of the bias estimate, i.e., the quantification of the available information about the biases in any scenario. Monte Carlo simulations show that this method has significant improvement of performance with reducing the RMS errors about $60-80 \%$ comparing to the commonly used decoupled Kalman filtering method. Furthermore, the new method is shown be statistically efficient, i.e., it meets the CRLB. The extension of the new technique for dynamically varying biases is also presented.
\end{abstract}

Keywords: Sensor bias estimation, registration, multisensor-multitarget tracking, decoupled Kalman filtering.

\section{Introduction}

Registration error correction is vital in multiple sensor systems in order to carry out data fusion. This requires estimation of the sensor measurement biases. It is important to correct for these bias errors so that the multiple sensor measurements and/or tracks can be referenced to a common tracking coordinate system (frame). If uncorrected, registration error can lead to large tracking errors and potentially to the formation of multiple tracks (ghosts) on the same target.

In this paper, we consider a multiple sensor tracking system with a decentralized information processing architecture. Each local tracker obtains its own local state estimates using local measurements and sends the estimates to the fusion center. The fusion center performs track-to-track fusion on demand after estimating these sensor biases based on the common targets being tracked by the different sensors (see, e.g, [5]).

To estimate the bias vector, the classical approach is to augment the system state to include the bias vector as part of the state, then implement an augmented state Kalman filter (ASKF) by stacking the state of all the targets and the sensor biases into a single vector. The problem with this approach is that the implementation of this ASKF can be computationally infeasible. In addition, numerical problems may arise during the implementation; mainly, for ill-conditioned systems. Friedland [9] proposed the idea of implementing two parallel, reduced-order filters instead of the use of a ASKF. Alouani, Rice and Blair [1] showed that under a restrictive algebraic constraint, the optimal estimate of the state can be obtained as a linear combination of the outputs of the local bias-ignorant estimate and the bias estimate. They claimed that since the algebraic constraint can be too restrictive in practice, that is an indirect proof why all practical two-stage filters are suboptimal. Van Doorn and Blom [18] gave the exact solution for the augmented Kalman filter problem but then decoupled the equations using an approximation in order to make the implementation feasible. A similar approach was used in $[10,16]$ to separate the tracker from the bias filter and it undoubtedly imposed some (uncharacterized) loss in estimation performance. The method of [17] deals only with translational biases and accomplishes only relative registration.

Lin, Kirubarajan and Bar-Shalom [13] presented the bias estimation based on the local track estimates at a single time, i.e., based on a single frame. The purpose of this paper is to extend the work of [13] to a bias model which models both the offset biases and the scale biases, and also includes the dynamic bias estimation based on the local track estimates at different times, 
i.e., based on multiple frames. This technique has been shown to yield absolute registration for biases that enter nonlinearly in the measurement equation subject to an observability condition.

This paper is organized as follows. The bias model and the assumptions for bias estimation are discussed in Section 2. In Section 3, we discuss the dynamic bias estimation, including the Recursive Least Squares (RLS) estimator and the optimal MMSE estimator of the bias. The decoupled Kalman filtering method [18] is also briefly described in this section. Section 4 presents the performance of the bias estimators comparing with the decoupled Kalman filtering for a typical scenario. It is shown that the proposed technique achieves, similarly to [13], absolute registration. Conclusions are in Section 5.

\section{Bias Model}

Consider $M$ static sensors which measure range and azimuth for all $N$ common targets in the surveillance region. The location of each sensor is assumed to be known exactly. The following model for biased measurements in polar coordinates is adopted for sensor $i$ :

$$
z_{i}^{p}(k)=\left[\begin{array}{c}
r_{i}(k) \\
\theta_{i}(k)
\end{array}\right]
$$

where range and azimuth at time $k$ are

$$
\begin{aligned}
r_{i}(k) & =\left[1+\epsilon_{i}^{r}(k)\right] r_{i}^{t}(k)+b_{i}^{r}(k)+w_{i}^{r}(k) \\
\theta_{i}(k) & =\left[1+\epsilon_{i}^{\theta}(k)\right] \theta_{i}^{t}(k)+b_{i}^{\theta}(k)+w_{i}^{\theta}(k)
\end{aligned}
$$

$r_{i}^{t}(k)$ and $\theta_{i}^{t}(k)$ are the true range and azimuth. The measurement noises $w_{i}^{r}(k)$ and $w_{i}^{\theta}(k)$ are zero-mean white measurement noise with corresponding variances $\sigma_{r}^{2}$ and $\sigma_{\theta}^{2}$ and mutually independent to each other.

Denote

$$
\begin{aligned}
& C_{i}(k) \triangleq\left[\begin{array}{cccc}
1 & 0 & r_{i}^{t}(k) & 0 \\
0 & 1 & 0 & \theta_{i}^{t}(k)
\end{array}\right] \\
& \beta_{i}(k) \triangleq\left[\begin{array}{c}
b_{i}^{r}(k) \\
b_{i}^{\theta}(k) \\
\epsilon_{i}^{r}(k) \\
\epsilon_{i}^{\theta}(k)
\end{array}\right]
\end{aligned}
$$

Then,

$$
z_{i}^{p}(k)=\left[\begin{array}{c}
r_{i}^{t}(k) \\
\theta_{i}^{t}(k)
\end{array}\right]+C_{i}(k) \beta_{i}(k)+\left[\begin{array}{c}
w_{i}^{r}(k) \\
w_{i}^{\theta}(k)
\end{array}\right]
$$

The observed (or estimated) azimuth $\hat{\theta}_{i}(k)$ and range $\hat{r}_{i}(k)$ can be used to obtain $C_{i}(k)$.

After transforming the measurements into Cartesian coordinates, the measurement equation for sensor $i$ is

$$
z_{i}(k)=H_{i}(k) \mathbf{x}(k)+B_{i}(k) C_{i}(k) \beta_{i}(k)+w_{i}(k)
$$

where the state vector $\mathbf{x}(k) \triangleq$ $\left[\begin{array}{llll}x(k) & \dot{x}(k) & y(k) & \dot{y}(k)\end{array}\right]^{\prime}, H_{i}(k)$ is the measurement matrix and $B_{i}(k)$ is a nonlinear function of the true range and azimuth. If $B_{i}(k)$ is constant, only the difference of the biases at different sensors is observable, which means "relative registration" or "incomplete observability". Using the observed (or estimated) azimuth $\hat{\theta}_{i}(k)$ and range $\hat{r}_{i}(k)$ from sensor $i$, one has the matrix $B_{i}(k)$ from $(7)$ as

$$
B_{i}(k)=\left[\begin{array}{cc}
\cos \hat{\theta}_{i}(k) & -\hat{r}_{i}(k) \sin \hat{\theta}_{i}(k) \\
\sin \hat{\theta}_{i}(k) & \hat{r}_{i}(k) \cos \hat{\theta}_{i}(k)
\end{array}\right]
$$

$w_{i}(k)$ is the measurement noise with the covariance in the Cartesian coordinates (omitting the time index $k$ in the measurements for simplicity)

$R_{i}(k)=\left[\begin{array}{cc}r_{i}^{2} \sigma_{\theta}^{2} \sin ^{2} \theta_{i}+\sigma_{r}^{2} \cos ^{2} \theta_{i} & \left(\sigma_{r}^{2}-r_{i}^{2} \sigma_{\theta}^{2}\right) \sin \theta_{i} \cos \theta_{i} \\ \left(\sigma_{r}^{2}-r_{i}^{2} \sigma_{\theta}^{2}\right) \sin \theta_{i} \cos \theta_{i} & r_{i}^{2} \sigma_{\theta}^{2} \cos ^{2} \theta_{i}+\sigma_{r}^{2} \sin ^{2} \theta_{i}\end{array}\right]$

\section{Dynamic Bias Estimation of Synchronous Sensors}

Consider a multiple sensor tracking system with a decentralized information processing architecture. Each local tracker obtains its own local state estimates using local measurements and sends the estimates to the fusion center. The fusion center estimate these sensor biases based on the common targets being tracked by the different sensors and then performs track-totrack fusion.

Assume the dynamic equation of the targets is

$$
\mathbf{x}(k+1)=F(k) \mathbf{x}(k)+v(k)
$$

where $F(k)$ is the transition matrix, and $v(k)$ is a zeromean white process noise with covariance $Q(k)$.

The local trackers have no bias information and can not estimate the sensor biases based on their own local measurements. Therefore, the local trackers have to assume there are no biases in the measurements and the local estimates are biased estimates. The model assumed by local trackers for a target (not indexed here, for simplicity) is

$$
\begin{aligned}
\mathbf{x}(k+1) & =F(k) \mathbf{x}(k)+v(k) \\
z_{i}(k) & =H_{i}(k) \mathbf{x}(k)+w_{i}(k)
\end{aligned}
$$

Note that, while the dynamic equation (11) is the same as (10), the measurement equation (12) is different from (7), with no bias term in equation (12). The local estimates are bias-ignorant state estimates ${ }^{1}$, and, consequently, there is a model mismatch problem.

\footnotetext{
${ }^{1}$ In the literature (e.g., [1]) these local estimators are called "bias-free", but this is a misnomer because they are biased but only the fusion center can estimate the biases.
} 


\subsection{The Pseudomeasurement of the Bias Vector}

Below, we will construct a pseudomeasurement based on these local bias-ignorant Kalman filters. We will derive the pseudomeasurement for the case of $M=2$ sensors. This can be easily extended to the case which $M$ is larger than two. Here we assume the bias to be an unknown constant.

From the Kalman filter of local tracker 1, we denote the filter gain and the residual as $W_{1}(k+1)$ and $\nu_{1}(k+$ 1), respectively. Then, we have

$$
\begin{aligned}
= & H_{2}(k+1) F(k) \mathbf{x}(k)+H_{2}(k+1) v(k)+ \\
& B_{2}(k+1) C_{2}(k+1) \beta_{2}(k+1)+ \\
& w_{2}(k+1)
\end{aligned}
$$

Note that the true state vector $\mathbf{x}(k)$ and the process noise $v(k)$ in equations (14) and (16) are same. Consequently, we define a pseudomeasurement of the bias vector as

$$
z_{b}(k+1) \triangleq z_{b}^{1}(k+1)-H_{1}(k+1) H_{2}^{\dagger}(k+1) z_{b}^{2}(k+1)
$$

Then,

$$
\begin{aligned}
\hat{\mathbf{x}}_{1}(k+1 \mid k+1)= & F(k) \hat{\mathbf{x}}_{1}(k \mid k)+W_{1}(k+1) \nu_{1}(k+1) \\
= & F(k) \hat{\mathbf{x}}_{1}(k \mid k)+W_{1}(k+1) \\
& {\left[z_{1}(k+1)-\hat{z}_{1}(k+1 \mid k)\right] } \\
= & F(k) \hat{\mathbf{x}}_{1}(k \mid k)+W_{1}(k+1) \\
& {\left[H_{1}(k+1) F(k) \mathbf{x}(k)+H_{1}(k+1)\right.} \\
& v(k)+B_{1}(k+1) C_{1}(k+1) \\
& \beta_{1}(k+1)+w_{1}(k+1)- \\
& \left.H_{1}(k+1) F(k) \hat{\mathbf{x}}(k \mid k)\right] \\
= & {\left[I-W_{1}(k+1) H_{1}(k+1)\right] F(k) } \\
& \hat{\mathbf{x}}_{1}(k \mid k)+W_{1}(k+1)\left[H_{1}(k+1)\right. \\
& F(k) \mathbf{x}(k)+H_{1}(k+1) v(k)+ \\
& B_{1}(k+1) C_{1}(k+1) \beta_{1}(k+1) \\
& \left.+w_{1}(k+1)\right]
\end{aligned}
$$

Note that the predicted measurement is based on the bias-ignorant measurement model assumed by the local tracker 1 , therefore there is no bias term in the predicted measurement.

Then, if we move the local state estimates to the left hand side of equation (13), and multiply on the left with the pseudoinverse of the gain ${ }^{2}$, we obtain

$$
\begin{aligned}
z_{b}^{1}(k+1) \triangleq & W_{1}^{\dagger}(k+1)\left[\hat{\mathbf{x}}_{1}(k+1 \mid k+1)-\right. \\
& \left.\left(I-W_{1}(k+1) H_{1}(k+1)\right) F(k) \hat{\mathbf{x}}_{1}(k \mid k)\right] \\
= & H_{1}(k+1) F(k) \mathbf{x}(k)+H_{1}(k+1) v(k)+ \\
& B_{1}(k+1) C_{1}(k+1) \beta_{1}(k+1)+ \\
& w_{1}(k+1)
\end{aligned}
$$

where the pseudoinverse of the gain is

$$
W_{i}^{\dagger} \triangleq\left(W_{i}^{\prime} W_{i}\right)^{-1} W_{i}^{\prime}
$$

Similarly, we define

$$
\begin{aligned}
z_{b}^{2}(k+1) \triangleq & W_{2}^{\dagger}(k+1)\left[\hat{\mathbf{x}}_{2}(k+1 \mid k+1)-\right. \\
& \left.\left(I-W_{2}(k+1) H_{2}(k+1)\right) F(k) \hat{\mathbf{x}}_{2}(k \mid k)\right]
\end{aligned}
$$

\footnotetext{
${ }^{2}$ Since the gain is, in general, not a square matrix, it is necessary to use the pseudoinverse.
}

$\begin{aligned} z_{b}(k+1)= & B_{1}(k+1) C_{1}(k+1) \beta_{1}(k+1)-H_{1}(k+1) \\ & H_{2}^{\dagger}(k+1) B_{2}(k+1) C_{2}(k+1) \beta_{2}(k+1)+ \\ & w_{1}(k+1)-H_{1}(k+1) H_{2}^{\dagger}(k+1) w_{2}(k+1)\end{aligned}$

Then, we have the pseudomeasurement equation of the bias vector

$$
z_{b}(k+1)=\mathcal{H}(k+1) \mathbf{b}(k+1)+\tilde{w}(k+1)
$$

where the pseudomeasurement matrix $\mathcal{H}(k+1)$, the bias parameter vector $\mathbf{b}(k+1)$ and the pseudomeasurement noise $\tilde{w}(k+1)$ are defined as

$$
\begin{aligned}
\mathcal{H}(k+1) \triangleq & {\left[B_{1}(k+1) C_{1}(k+1),\right.} \\
& \left.-H_{1}(k+1) H_{2}^{\dagger}(k+1) B_{2}(k+1) C_{2}(k+1)\right]
\end{aligned}
$$

$$
\begin{aligned}
& \mathbf{b}(k+1) \triangleq\left[\begin{array}{l}
\beta_{1}(k+1) \\
\beta_{2}(k+1)
\end{array}\right] \\
& \tilde{w}(k+1) \triangleq w_{1}(k+1)-H_{1}(k+1) H_{2}^{\dagger}(k+1) w_{2}(k+1)
\end{aligned}
$$

The bias pseudomeasurement noises $\tilde{w}$ are zero-mean and white, with covariance

$$
\begin{aligned}
\mathcal{R}(k+1)= & R_{1}(k+1)+H_{1}(k+1) H_{2}^{\dagger}(k+1) R_{2}(k+1) \\
& {\left[H_{1}(k+1) H_{2}^{\dagger}(k+1)\right]^{\prime} }
\end{aligned}
$$

The whiteness property of (22) is the key to the exact solution for the bias estimate - no approximations are needed. Note that no approximations whatsoever were made in deriving (19)-(23), i.e., this method, unlike $[18,10,16]$, is exact.

As a special case of the above, where the measurement matrices $H_{i}(k)$ are same for different local trackers, we have the following simplifications:

$$
\begin{aligned}
z_{b}(k+1)= & z_{b}^{1}(k+1)-z_{b}^{2}(k+1) \\
\mathcal{H}(k+1)= & {\left[B_{1}(k+1) C_{1}(k+1),\right.} \\
& \left.-B_{2}(k+1) C_{2}(k+1)\right] \\
\tilde{w}(k+1)= & w_{1}(k+1)-w_{2}(k+1)
\end{aligned}
$$


with the bias pseudomeasurement noises variance

$$
\mathcal{R}(k+1)=R_{1}(k+1)+R_{2}(k+1)
$$

\subsection{The Recursive Least Squares Bias Estimator}

If the bias parameter vector $\mathbf{b}(k)$ is modeled as an unknown constant, we can obtain the Recursive Least Squares (RLS) estimator based on the pseudomeasurement equation (19). The RLS estimator of the bias can obtain the estimate of the bias recursively, and the recursion is performed at two different levels. The first level is to improve the bias estimation recursively between different targets at a single time, i.e., based on a single frame. The second level is the recursion across multiple frames.

Assume the bias parameter estimate $\hat{\mathbf{b}}_{t-1}(k)$ and its corresponding estimation covariance $\Sigma_{t-1}(k)$ at time $k$ based on the first $t-1$ targets (pairs of tracks, since we assumed $M=2$ sensors) at time $k$ (and all the targets prior to $k$ ) are available, the recursive least squares (RLS) method can be implemented as follows to update the bias estimates at time $k$.

At time $k$, for each target $t=1, \ldots, N$ :

1. Get the new pseudomeasurement $z_{b, t}(k)=\mathcal{H}_{t}(k) \mathbf{b}+\tilde{w}_{t}(k)$, the measurement matrix $\mathcal{H}_{t}(k)$ using $(20)$ and the measurement noise covariance $\mathcal{R}_{t}(k)$ using (23).

2. Compute the bias update gain and the residual

$$
\begin{aligned}
G_{t}(k)= & \Sigma_{t-1}(k) \mathcal{H}_{t}(k)^{\prime}\left[\mathcal{H}_{t}(k) \Sigma_{t-1}(k) \mathcal{H}_{t}(k)^{\prime}+\right. \\
& \left.\mathcal{R}_{t}(k)\right]^{-1} \\
r_{t}(k)= & z_{b, t}(k)-\mathcal{H}_{t}(k) \hat{\mathbf{b}}_{t-1}(k)
\end{aligned}
$$

3. Update the bias estimate and its covariance

$$
\begin{aligned}
\hat{\mathbf{b}}_{t}(k)= & \hat{\mathbf{b}}_{t-1}(k)+G_{t}(k) r_{t}(k) \\
\Sigma_{t}(k)= & \Sigma_{t-1}(k)-\Sigma_{t-1}(k) \mathcal{H}_{t}(k)^{\prime}\left[\mathcal{H}_{t}(k) \Sigma_{t-1}(k)\right. \\
& \left.\mathcal{H}_{t}(k)^{\prime}+\mathcal{R}_{t}(k)\right]^{-1} \mathcal{H}_{t}(k) \Sigma_{t-1}(k)
\end{aligned}
$$

Note that the covariance update equation (31) may cause $\Sigma_{t}(k)$ to become indefinite due to round-off errors. We can use Joseph's form of the covariance update, namely,

$$
\begin{aligned}
\Sigma_{t}(k)= & {\left[I-G_{t}(k) \mathcal{H}_{t}(k)\right] \Sigma_{t-1}(k) } \\
& {\left[I-G_{t}(k) \mathcal{H}_{t}(k)\right]^{\prime}+G_{t}(k) G_{t}(k)^{\prime} }
\end{aligned}
$$

When the update with the last pseudomeasurement is completed, one has

$$
\begin{aligned}
\hat{\mathbf{b}}_{0}(k+1) & \triangleq \hat{\mathbf{b}}_{N}(k) \\
\Sigma_{0}(k+1) & \triangleq \Sigma_{N}(k)
\end{aligned}
$$

\subsection{The Optimal MMSE Estimator of a Time-Varying Bias}

If the bias is modeled as a random sequence, under the Gaussian assumption we can obtain the optimal MMSE estimator based on the pseudomeasurement equation (19) and the dynamic equation of the bias.

Assume the dynamic equation of the stacked bias parameter vector is

$$
\mathbf{b}(k+1)=F_{b}(k) \mathbf{b}(k)+v_{b}(k)
$$

where $F_{b}(k)$ is the transition matrix of the bias vector $\mathbf{b}$, and $v_{b}(k)$ is the stacked process noise of the bias vector, zero-mean white with covariance $Q_{b}(k)$.

Assume the bias estimate $\hat{\mathbf{b}}_{t-1}(k \mid k)$ and its corresponding estimation covariance $\Sigma_{t-1}(k \mid k)$ at time $k$ based on the first $t-1$ targets at time $k$ (and all the targets prior to $k$ ) are available. A Kalman filter can then be used to update the bias parameter estimate and the corresponding covariance recursively at time $k$ as follows.

At time $k$, for each target $t=1, \ldots, N$ :

1. Get the new pseudomeasurement $z_{b, t}(k)=\mathcal{H}_{t}(k) \mathbf{b}(k)+\tilde{w}_{t}(k)$, the measurement matrix $\mathcal{H}_{t}(k)$ using $(20)$ and the measurement noise covariance $\mathcal{R}_{t}(k)$ using (23).

2. Compute the bias update gain and the residual

$$
\begin{aligned}
G_{t}(k)= & \Sigma_{t-1}(k \mid k) \mathcal{H}_{t}(k)^{\prime}\left[\mathcal{H}_{t}(k) \Sigma_{t-1}(k \mid k) \mathcal{H}_{t}(k)^{\prime}\right. \\
& \left.+\mathcal{R}_{t}(k)\right]^{-1} \\
r_{t}(k)= & z_{b_{t}}(k)-\mathcal{H}_{t}(k) \hat{\mathbf{b}}_{t-1}(k \mid k)
\end{aligned}
$$

3. Update the bias estimate and its covariance

$$
\begin{array}{rlr}
\hat{\mathbf{b}}_{t}(k \mid k)= & \hat{\mathbf{b}}_{t-1}(k \mid k)+G_{t}(k) r_{t}(k) \\
\Sigma_{t}(k \mid k)= & \Sigma_{t-1}(k \mid k)-\Sigma_{t-1}(k \mid k) \mathcal{H}_{t}(k)^{\prime}\left[\mathcal{H}_{t}(k)\right. \\
& \left.\Sigma_{t-1}(k \mid k) \mathcal{H}_{t}(k)^{\prime}+\mathcal{R}_{t}(k)\right]^{-1} \mathcal{H}_{t}(k) \\
& \Sigma_{t-1}(k \mid k)
\end{array}
$$

or, instead of (39), using Joseph's form for the covariance update

$$
\begin{aligned}
\Sigma_{t}(k \mid k)= & {\left[I-G_{t}(k) \mathcal{H}_{t}(k)\right] \Sigma_{t-1}(k \mid k) } \\
& {\left[I-G_{t}(k) \mathcal{H}_{t}(k)\right]^{\prime}+G_{t}(k) G_{t}(k)^{\prime} }
\end{aligned}
$$

When the update with the last pseudomeasurement is completed, the predicted biases and covariances at time $k+1$ are

$$
\begin{aligned}
\hat{\mathbf{b}}(k+1 \mid k) & =F_{b}(k) \hat{\mathbf{b}}_{N}(k \mid k) \\
\Sigma(k+1 \mid k) & =F_{b}(k) \Sigma_{N}(k \mid k) F_{b}(k)^{\prime}+Q_{b}(k)
\end{aligned}
$$


Then, one has, for the start of the next cycle,

$$
\begin{aligned}
\hat{\mathbf{b}}_{0}(k+1 \mid k+1) & \triangleq \hat{\mathbf{b}}(k+1 \mid k) \\
\Sigma_{0}(k+1 \mid k+1) & \triangleq \Sigma(k+1 \mid k)
\end{aligned}
$$

\subsection{The Decoupled Kalman Filtering}

The bias estimation is coupled with the target state estimations. In order to avoid an augment state Kalman filter, which can be computational infeasible, the decoupled Kalman filtering is commonly used, as in $[18,10,16]$. The decoupled Kalman filtering used in [18] consists of the following three steps when the bias is modeled by the dynamic equation (35).

1. Bias prediction

Assume the bias estimate $\hat{\mathbf{b}}(k \mid k)$ and the corresponding estimation covariance $\Sigma(k \mid k)$ at time $k$ are available. The predicted bias and the corresponding covariance at time $k+1$ are

$$
\begin{aligned}
\hat{\mathbf{b}}(k+1 \mid k) & =F_{b}(k) \hat{\mathbf{b}}(k \mid k) \\
\Sigma(k+1 \mid k) & =F_{b}(k) \Sigma(k \mid k) F_{b}(k)^{\prime}+Q_{b}(k)
\end{aligned}
$$

2. State estimate and covariance update for targets For target $t=1, \ldots, N$, (omitting the index $t$ for simplicity), the predicted state estimate, its covariance and the predicted measurements are

$$
\begin{aligned}
\hat{\mathbf{x}}(k+1 \mid k)= & F(k) \hat{\mathbf{x}}(k \mid k) \\
P(k+1 \mid k)= & F(k) P(k \mid k) F(k)^{\prime}+Q(k) \\
\hat{\mathbf{z}}(k+1 \mid k)= & \mathbf{H}(k+1) \hat{\mathbf{x}}(k+1 \mid k)+ \\
& \mathbf{H}_{b}(k+1) \hat{\mathbf{b}}(k+1 \mid k)
\end{aligned}
$$

where, when assuming $M=2$ sensors,

$$
\begin{aligned}
\hat{\mathbf{z}}(k+1 \mid k) & \triangleq\left[\begin{array}{c}
\hat{z}_{1}(k+1 \mid k) \\
\hat{z}_{2}(k+1 \mid k)
\end{array}\right] \\
\mathbf{H}(k) & \triangleq\left[\begin{array}{c}
H_{1}(k) \\
H_{2}(k)
\end{array}\right] \\
\mathbf{H}_{b}(k) & \triangleq\left[\begin{array}{cc}
B_{1}(k) C_{1}(k) & \mathbf{0} \\
\mathbf{0} & B_{2}(k) C_{2}(k)
\end{array}\right]
\end{aligned}
$$

The covariance between the states and the measurements, and the measurement prediction covariance at time $k+1$ are

$$
\begin{aligned}
P_{\mathbf{x} \mathbf{z}}(k+1 \mid k)= & P(k+1 \mid k) \mathbf{H}(k+1)^{\prime}+ \\
& P_{\mathbf{x b}}(k+1 \mid k) \mathbf{H}_{b}(k+1)^{\prime}
\end{aligned}
$$

$$
\begin{aligned}
P_{\mathbf{z z}}(k+1)= & \mathbf{H}(k+1) P(k+1 \mid k) \mathbf{H}(k+1)^{\prime}+ \\
& \mathbf{H}_{b}(k+1) P_{\mathbf{b x}}(k+1 \mid k) \mathbf{H}(k+1)^{\prime} \\
& +\mathbf{H}(k+1) P_{\mathbf{x b}}(k+1 \mid k) \mathbf{H}_{b}(k+1)^{\prime} \\
& +\mathbf{H}_{b}(k+1) \Sigma(k+1 \mid k) \mathbf{H}_{b}(k+1)^{\prime} \\
& +\mathbf{R}(k+1)
\end{aligned}
$$

where $P_{\mathbf{b x}}=P_{\mathbf{x b}}^{\prime}$ is the crosscovariance between the states and the biases, and the measurement noise covariance is

$$
\mathbf{R}(k+1)=\left[\begin{array}{cc}
R_{1}(k+1) & \mathbf{0} \\
\mathbf{0} & R_{2}(k+1)
\end{array}\right]
$$

In order to decouple the state estimation and the bias estimation, two approximations are used in [18] to simplify (53) and (54) and achieve decoupling between the target state estimation and the bias estimation. Two simplifying assumptions are made in [18]: in the target state estimation, one assumes that the bias covariance is zero, i.e.,

$$
\Sigma(\cdot)=0
$$

and (consequently)

$$
P_{\mathbf{x b}}(\cdot)=0
$$

Then one has the VDB technique [18]

$$
\begin{aligned}
P_{\mathbf{x z}}(k+1 \mid k) \approx & P(k+1 \mid k) \mathbf{H}(k+1)(k+1)^{\prime} \\
& \triangleq \tilde{\tilde{P}}_{\mathbf{x z}}(k+1 \mid k) \\
P_{\mathbf{z z}}(k+1) \approx & \mathbf{H}(k+1) P(k+1 \mid k) \mathbf{H}(k+1)^{\prime} \\
& +\mathbf{R}(k+1) \triangleq \tilde{\tilde{P}}_{\mathbf{z z}}(k+1)
\end{aligned}
$$

In our simulation scenario, we experienced divergences in some runs using (58)-(59). Consequently we modified it by making one assumption less: only the crosscovariance (57) is assumed zero but $\Sigma(\cdot)$ is not assumed zero.

Then one has the modified VDB technique

$$
\begin{aligned}
\tilde{P}_{\mathbf{x z}}(k+1 \mid k) \approx & P(k+1 \mid k) \mathbf{H}(k+1)^{\prime} \\
& =\tilde{\tilde{P}}_{\mathbf{z z}}(k+1 \mid k) \\
\tilde{P}_{\mathbf{z z}}(k+1) \approx & \mathbf{H}(k+1) P(k+1 \mid k) \mathbf{H}(k+1)^{\prime}+ \\
& \mathbf{H}_{b}(k+1) \Sigma(k+1 \mid k) \mathbf{H}_{b}(k+1)^{\prime} \\
& +\mathbf{R}(k+1) \neq \tilde{\tilde{P}}_{\mathbf{z z}}(k+1)
\end{aligned}
$$

The updated state estimates and the updated covariances are computed as

$$
\begin{aligned}
\hat{\mathbf{x}}(k+1 \mid k+1)= & \mathbf{x}(k+1 \mid k)+\tilde{P}_{\mathbf{x z}}(k+1 \mid k) \\
& \tilde{P}_{\mathbf{z z}}(k+1)^{-1}[\mathbf{z}(k+1)-
\end{aligned}
$$




$$
P(k+1 \mid k+1)=\begin{aligned}
& \hat{\mathbf{z}}(k+1 \mid k)] \\
& P(k+1 \mid k)-\tilde{P}_{\mathbf{x z}}(k+1 \mid k) \\
& \tilde{P}_{\mathbf{z z}}(k+1)^{-1} \tilde{P}_{\mathbf{x z}}(k+1 \mid k)^{\prime}
\end{aligned}
$$

for the modified VDB technique, or with the double tilde for the original VDB technique.

3. Bias estimates and covariances update

The updated state estimates of the targets can be applied to update the bias parameter estimate and the corresponding covariance. This can be performed recursively between the different targets since the measurement noises for the different targets are mutually uncorrelated.

Assume the bias parameter estimate $\hat{\mathbf{b}}_{t-1}(k+1 \mid k+$ 1) and the corresponding covariance $\Sigma_{t-1}(k+1 \mid k+$ 1) at time $k+1$ based on the first $t-1$ targets at time $k+1$ (and all the targets prior to $k+1$ ) are available. A Kalman filter is used to update the bias parameter estimate and the corresponding covariance recursively at time $k+1$ as

$$
\begin{aligned}
\hat{\mathbf{b}}_{t}(k+1 \mid k+1)= & \hat{\mathbf{b}}_{t-1}(k+1 \mid k+1)+ \\
& P_{\mathbf{b z}, t}(k+1) \tilde{P}_{\mathbf{z z}, t}(k+1)^{-1} \\
& {\left[\mathbf{z}_{t}(k+1)-\mathbf{H}_{t}(k+1)\right.} \\
& \hat{\mathbf{x}}_{t}(k+1 \mid k+1)-\mathbf{H}_{b, t}(k+1) \\
& \left.\hat{\mathbf{b}}_{t-1}(k+1 \mid k+1)\right] \quad(64) \\
\Sigma_{t}(k+1 \mid k+1)= & \Sigma_{t-1}(k+1 \mid k+1)- \\
& P_{\mathbf{b z}, t}(k+1) \tilde{P}_{\mathbf{z z}, t}(k+1)^{-1} \\
& P_{\mathbf{b z}, t}(k+1)^{\prime}
\end{aligned}
$$

where the approximation of $P_{\mathbf{b z}, t}(k+1)$ is [18]

$$
P_{\mathbf{b z}, t}(k+1) \approx \Sigma_{t-1}(k+1 \mid k+1) \mathbf{H}_{b, t}(k+1)^{\prime}
$$

in view of (57).

When the bias update with the last target is completed $^{3}$, one has

$$
\begin{aligned}
\hat{\mathbf{b}}(k+1 \mid k+1) & \triangleq \hat{\mathbf{b}}_{N}(k+1 \mid k+1) \\
\Sigma(k+1 \mid k+1) & \triangleq \Sigma_{N}(k+1 \mid k+1)
\end{aligned}
$$

If the bias is modeled as an unknown constant, this can be viewed as a special case of the decoupled Kalman filtering described above with $F_{b_{i}}=1$ and $Q_{b_{i}}=\mathbf{0}$.

\footnotetext{
${ }^{3}$ If the measurements from the different targets within the same frame have different time stamps, then they are just used sequentially for bias estimation.
}

Therefore, the bias prediction step is not necessary. The other two steps, the state estimate update step and the bias estimate update step, can be easily obtained.

\section{Simulation Results}

Consider an example with 2 sensors and 32 targets. Assume that the sensors are located $100 \mathrm{~km}$ apart and perfectly synchronized. The biases for the two sensors are

$$
\beta_{1}=\beta_{2}=\left[\begin{array}{llll}
20 \mathrm{~m} & 2 \mathrm{mrad} & 10^{-4} & 10^{-3}
\end{array}\right]^{\prime}
$$

The standard deviation of the measurement noise variances are $\sigma_{r}=10 \mathrm{~m}$ and $\sigma_{\theta}=1 \mathrm{mrad}$ for the range and the azimuth measurements, respectively.

The dynamics of the target are modeled using Discretized Continuous White Noise Acceleration (DCWNA) models [7] where the transition matrix $F(k)$ in the dynamic equation (10) is defined as

$$
F(k) \triangleq\left[\begin{array}{cccc}
1 & T & 0 & 0 \\
0 & 1 & 0 & 0 \\
0 & 0 & 1 & T \\
0 & 0 & 0 & 1
\end{array}\right]
$$

$T$ is the sampling interval, and the process noise covariance

$$
\begin{aligned}
Q & =\left[\begin{array}{cc}
Q_{x} & 0 \\
0 & Q_{y}
\end{array}\right] \\
Q_{x} & =\left[\begin{array}{cc}
\frac{1}{3} T^{3} & \frac{1}{2} T^{2} \\
\frac{1}{2} T^{2} & T
\end{array}\right] \tilde{q}_{x} \\
Q_{y} & =\left[\begin{array}{cc}
\frac{1}{3} T^{3} & \frac{1}{2} T^{2} \\
\frac{1}{2} T^{2} & T
\end{array}\right] \tilde{q}_{y}
\end{aligned}
$$

with the power spectral densities $\tilde{q}_{x}=\tilde{q}_{y}=6 \mathrm{~m}^{2} / \mathrm{s}^{3}$. The measurement matrix $H_{i}(k)$ is

$$
H_{i}(k)=\left[\begin{array}{cccc}
1 & 0 & 0 & 0 \\
0 & 0 & 1 & 0
\end{array}\right]
$$

The geometry of the targets is shown in Figure 1 and targets are moving at nearly constant velocity with $v_{x}=v_{y}=20 \mathrm{~m} / \mathrm{s}$. The sampling intervals are $T=1 \mathrm{~s}$. The initial state estimates are generated randomly with $\mathcal{N}\left(\mathbf{x}_{t}, P(0 \mid 0)\right)$ where $P(0 \mid 0)=$ $\operatorname{diag}\left[(200 \mathrm{~m})^{2},(20 \mathrm{~m} / \mathrm{s})^{2},(200 \mathrm{~m})^{2},(20 \mathrm{~m} / \mathrm{s})^{2}\right]$ and $\mathbf{x}_{t}$ is the true state. The initial bias parameter estimate of sensor $i$ is zero with the initial bias covariance $\Sigma_{i}(0 \mid 0)=$ $\operatorname{diag}\left[(100 \mathrm{~m})^{2},(30 \mathrm{mrad})^{2},(0.005)^{2},(0.002)^{2}\right]$.

We compare the RMS errors of the exact method with the modified VDB based on 100 Monte Carlo 


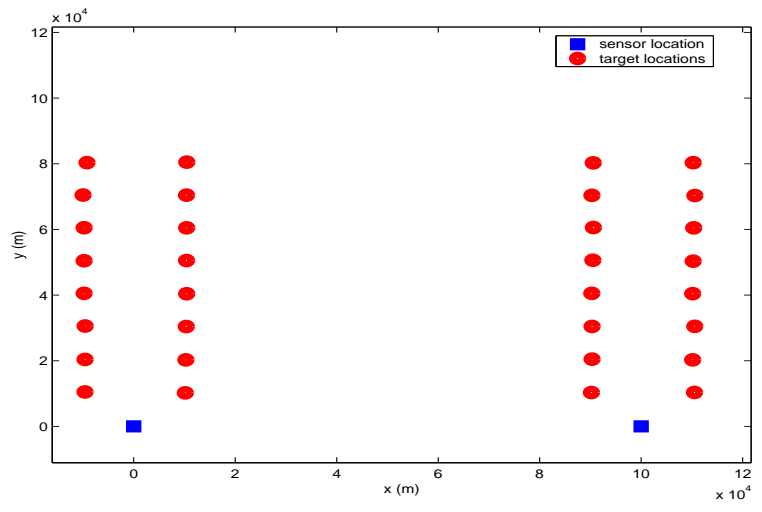

Figure 1: The geometry of the targets and the sensors

runs. The range offset RMS errors are shown in Figure 2 and the significant improvement on RMS errors can be seen in the figure, with less than $5 \mathrm{~m}$ range offset RMS bias errors for the exact method at step $k=20$ vs. about $12 \mathrm{~m}$ for the modified VDB method, which means the exact method reduced the bias errors about $60 \%$. Similar significant improvement in performance can also be seen in the RMS azimuth offset bias errors shown in Figure 3 with about $0.1 \mathrm{mrad}$ azimuth RMS errors for the exact method at step $k=20$ vs. up to $1.5 \mathrm{mrad}$ for the modified VDB method. For the modified VDB method, the RMS estimation error tends to a constant - after a short time it does not improve any further, unlike in the case of the exact method.

The sensor's azimuth bias (which is equivalent to crossrange bias) is reduced by the other sensor's range measurements and we see a significant reduction to about $1 / 10$ of the azimuth standard deviation. One sensor's range bias is reduced by the other sensors crossrange measurements. Since the crossrange standard deviation is around $100 \mathrm{~km} \times 1 \mathrm{mrad}=100 \mathrm{~m}$, it is difficult to achieve a significant reduction. Nevertheless the exact algorithm reduced the range bias to about $1 / 2$ of the range standard deviation.

The range and azimuth scale RMS errors are shown in Figures 4 and 5. The significant improvements on RMS errors can be seen in these figures, with about $6 \times 10^{-5}$ range scale RMS errors for the exact method at step $k=20$ vs. about $25 \times 10^{-5}$ for the modified VDB method. Similar improvement can also be seen in the azimuth scale RMS errors with about $1 \times 10^{-4}$ azimuth scale RMS errors for the exact method at step $k=20$ vs. $5 \times 10^{-4}$ for the modified VDB method.

The Normalized Estimation Error Squared (NEES) and the $95 \%$ probability interval of the exact method is shown in Figure 6 and it can be seen to be in its acceptance region for every step $k$, which means the estimator is consistent. The NEES of the modified VDB

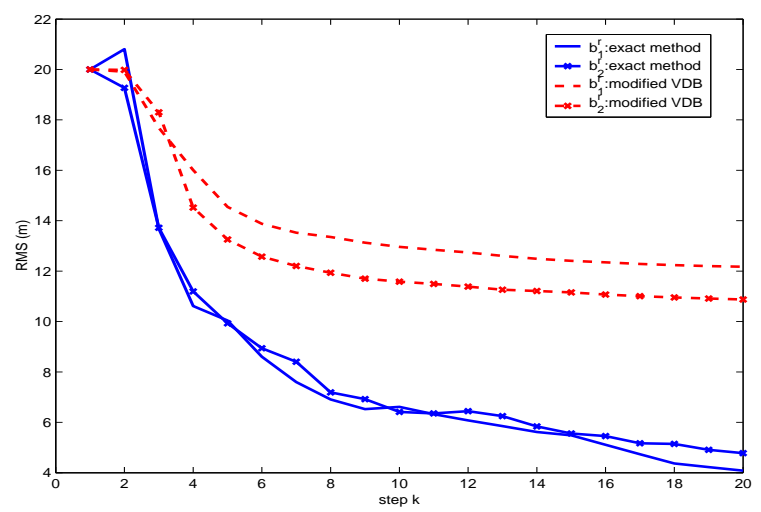

Figure 2: Range offset RMS errors

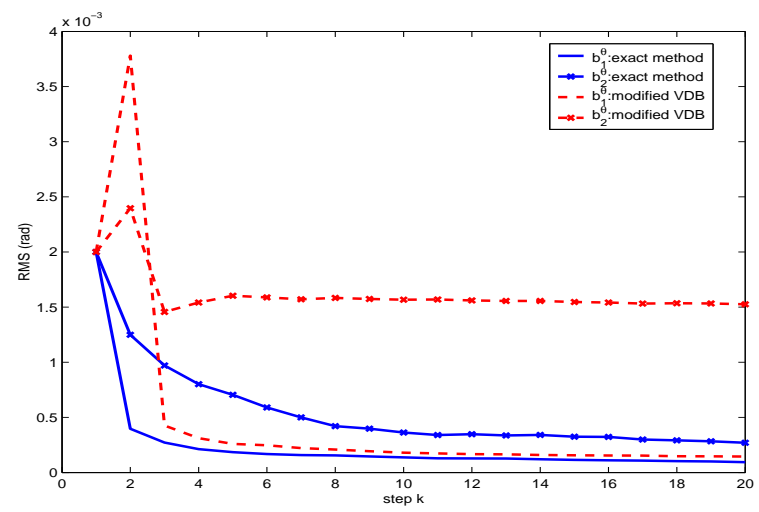

Figure 3: Azimuth offset RMS errors

method diverges because of its RMS error tending to a constant while its calculated variance goes to zero. Figures 7-10 compare the RMS errors of the exact method for sensor 1 with the square root of the diagonal elements of the Cramer-Rao Lower Bound (CRLB). It is clear that the RMS errors achieve the CRLB, which means the estimator is efficient.

\section{Conclusions}

In this paper we provide the exact solution for the multisensor bias estimation problem based on local tracks. It is shown that the bias estimate can be obtained dynamically based on the (biased) outputs of the local state estimators. This is accomplished by manipulating the local state estimates such that they yield pseudomeasurements of the sensor biases with additive noises that are zero-mean, white and with easily calculated covariances.

The main advantages and features of the exact method can be summarized as follows: (1) the exact method works with arbitrary number of sensors in arbitrary locations; (2) the solution is exact, no approximation has been made; (3) the algorithm is 


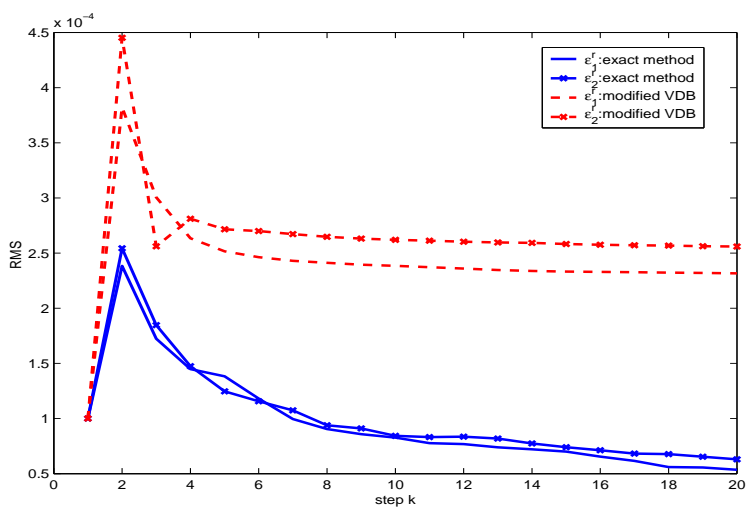

Figure 4: Range scale RMS errors

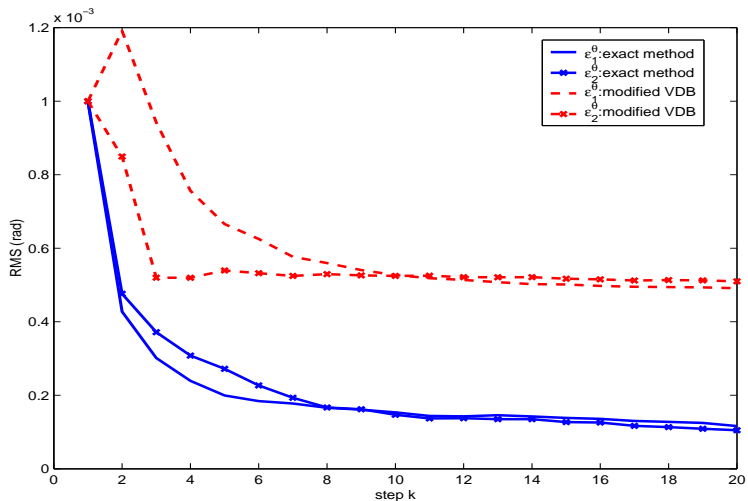

Figure 5: Azimuth scale RMS errors

implemented recursively which is computational inexpensive; (4) these results enable the evaluation of the Cramer-Rao Lower Bound on the covariance of the bias estimate for a constant bias, i.e., the quantification of the available information about the biases in any scenario and the CRLB is achievable. The exact method has also been shown to be statistically efficient.

Monte Carlo simulations show that this exact method yields significant improvements in performance with reduced RMS errors up to about $60-80 \%$ compared with the commonly used decoupled Kalman filtering method. The RMS errors are also shown to be consistent with the theoretical CRLB for sensor registration. This algorithm is also adapted for timevarying biases with similar results.

\section{References}

[1] Alouani, A. T., Rice, T. R., and Blair, W. D., "A Two-Stage Filter for State Estimation in the Presence of Dynamical Stochastic Bias", Proc. 1992 American Control Conf., June 1992, 1784-1788.

[2] Bar-Shalom, Y., "Airborne GMTI Radar Position Bias Estimation Using Static-Rotator Targets

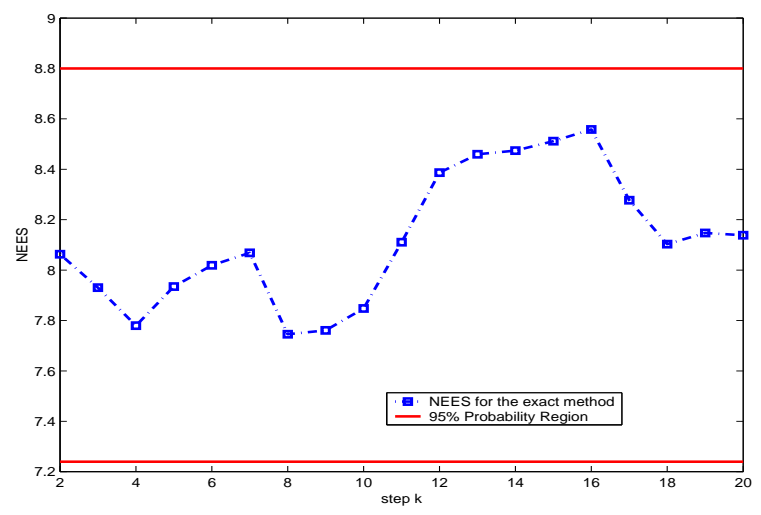

Figure 6: NEES for the exact method

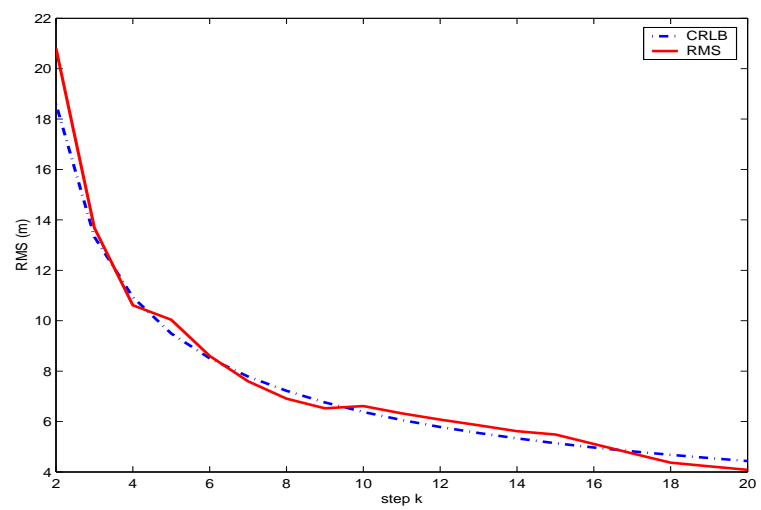

Figure 7: Range offset RMS error of the exact method and the square root of CRLB

of Opportunity", IEEE Trans. Aerosp. Electronic Systems, Vol. 37, No.2, 695-699, April 2001.

[3] Bar-Shalom, Y., (editor), Multitarget-Multisensor Tracking: Advanced Applications, Artech House, 1990 (reprinted by YBS Publishing, 1998).

[4] Bar-Shalom, Y., (editor), Multitarget-Multisensor Tracking: Applications and Advances, Vol. II, Artech House, 1992 (reprinted by YBS Publishing, 1998).

[5] Bar-Shalom, Y. and Li, X. R., MultitargetMultisensor Tracking: Principles and Techniques, YBS Publishing, 1995.

[6] Bar-Shalom, Y. and Blair, W. D., (editors), Multitarget-Multisensor Tracking: Applications and Advances, Vol. III, Artech House, 2000.

[7] Bar-Shalom, Y., Li, X. R., and Kirubarajan, T., Estimation with Applications to Tracking and Navigation: Algorithms and Software for Information Extraction, Wiley, 2001. 


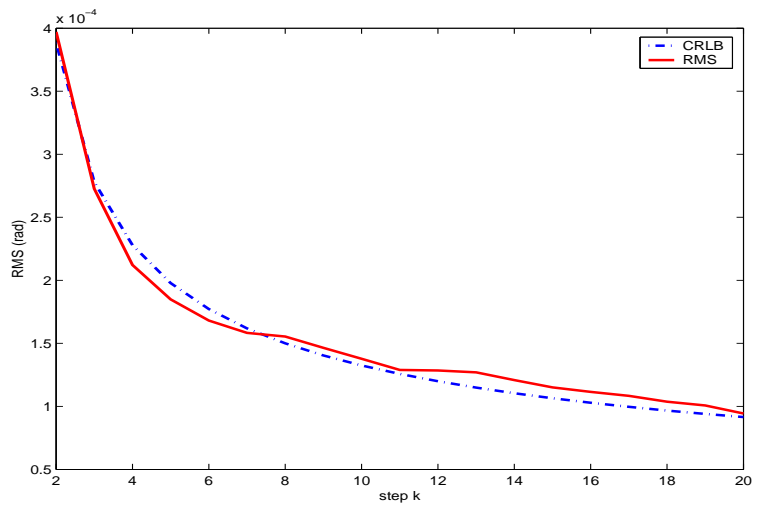

Figure 8: Azimuth offset RMS error of the exact method and the square root of CRLB

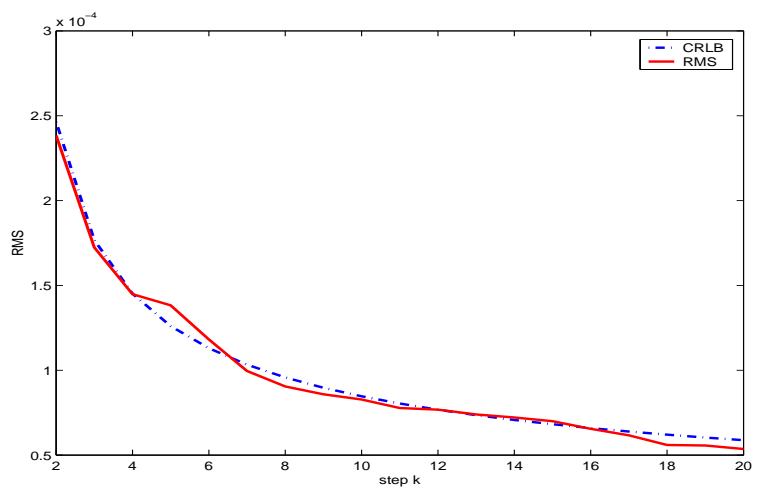

Figure 9: Range scale RMS error of the exact method and the square root of CRLB

[8] Blackman, S. S., and Popoli, R., Design and Analysis of Modern Tracking Systems, Dedham, MA: Artech House, 1999.

[9] Freidland, B., "Treatment of Bias in Recursive Filtering", IEEE Trans. Auto. Cont.,, Vol. AC14, August 1969.

[10] Kastella, K., et al., "Bias Modeling and Estimation for GMTI Applications", In Proceedings of the 3rd International Conference on Information Fusion, Paris, France, July 2000.

[11] Kenefic, R. J., "Local and Remote Track File Registration Using Minimum Description Length", IEEE Trans. Aerosp. Electronic Systems, 29(3):651-655, July 1993.

[12] Kirubarajan, T., and Bar-Shalom, Y., "Low Observable Target Motion Analysis Using Amplitude Information", IEEE Trans. Aerosp. Electronic Systems, 32(4):1367-1384, October 1996.

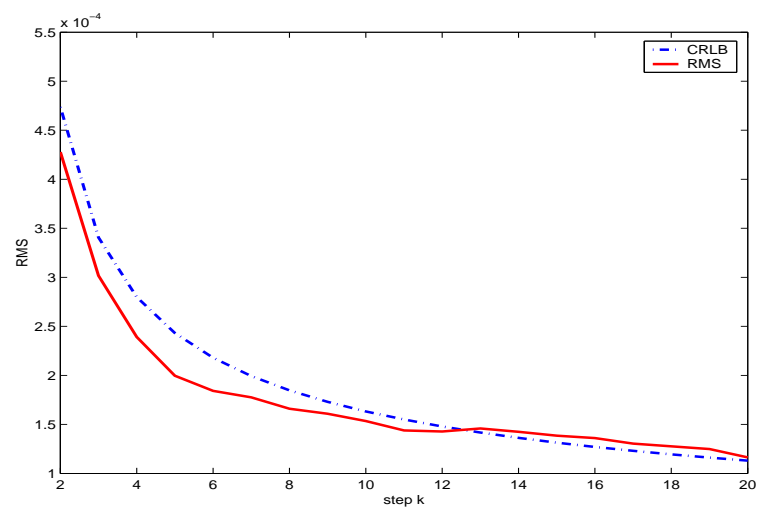

Figure 10: Azimuth scale RMS error of the exact method and the square root of CRLB

[13] Lin, X., Kirubarajan, T., and Bar-Shalom, Y., "Multisensor Bias Estimation with Local Tracks Without a Priori Association", Proc. SPIE, August 2003 .

[14] S. Mori, K. C. Chang, C. Y. Chong, K. P. Dunn, "Tracking Performance Evaluation — Track Accuracy in Dense Target Environments", in Proc. SPIE 1990 Tech. Symp. on Aerospace Sensing, Orlando, FL, April 1990.

[15] Pattipati, K. R., Popp, R. L, and Kirubarajan, T., "Survey of Assignment Techniques for Multitarget Tracking", Chapter 2 in Bar-Shalom, Y. and Blair, W. D. (eds), Mutlitarget-Multisensor Tracking: Applications and Advances, Volume III, Artech House, 2000.

[16] Shea, P. J., et al., "Precision Tracking of Ground Targets", Proceedings of the IEEE Aerospace Conference, Big Sky, MT, March 2000.

[17] Stone, L. D., Williams, M., and Tran, T., "Trackto-Track Association and Bias Removal", Proc. SPIE, Vol.4728, 2002.

[18] van Doorn, B. A. and Blom, H. A. P., "Systematic Error Estimation in Multisensor Fusion Systems", Proc. SPIE, Vol.1954, April 1993. 